\title{
First observations of magnetic holes deep within the coma of a comet
}

\author{
F. Plaschke ${ }^{1}$, T. Karlsson ${ }^{2,1}$, C. Götz ${ }^{3}$, C. Möstl ${ }^{1}$, I. Richter ${ }^{3}$, M. Volwerk ${ }^{1}$, A. Eriksson ${ }^{4}$, E. Behar ${ }^{5}$, and R. Goldstein ${ }^{6}$ \\ ${ }^{1}$ Space Research Institute, Austrian Academy of Sciences, Schmiedlstrasse 6, 8042 Graz, Austria \\ e-mail: ferdinand.plaschke@oeaw.ac.at \\ 2 Department of Space and Plasma Physics, School of Electrical Engineering and Computer Science, Royal Institute of Technology, \\ Stockholm, Sweden \\ ${ }^{3}$ Institute of Geophysics and Extraterrestrial Physics, Braunschweig Institute of Technology, Braunschweig, Germany \\ ${ }^{4}$ Swedish Institute of Space Physics, Uppsala, Sweden \\ ${ }^{5}$ Swedish Institute of Space Physics, Kiruna, Sweden \\ ${ }^{6}$ Southwest Research Institute, San Antonio, USA
}

Received 25 April 2018 / Accepted 5 July 2018

\begin{abstract}
The Rosetta spacecraft of the European Space Agency made ground-breaking observations of comet 67P/Churyumov-Gerasimenko and of its cometary environment. We search for magnetic holes in that environment, i.e., significant depressions in the magnetic field strength, measured by the Rosetta fluxgate Magnetometer (MAG) in April and May 2015. In that time frame of two months, we identified 23 magnetic holes. The cometary activity was intermediate and increasing because Rosetta was on the inbound leg toward the Sun. While in April solar wind protons were still observed by Rosetta near the comet, in May these protons were already mostly replaced by heavy cometary ions. Magnetic holes have frequently been observed in the solar wind. We find, for the first time, that magnetic holes exist in the cometary environment even when solar wind protons are almost absent. Some of the properties of the magnetic holes are comparable to those of solar wind holes; they are associated with density enhancements, sometimes associated with co-located current sheets and fast solar wind streams, and are of similar scales. However, particularly in May, the magnetic holes near the comet appear to be more processed, featuring shifted density enhancements and, sometimes, bipolar signatures in magnetic field strength rather than simple depressions. The magnetic holes are of global size with respect to the coma. However, at the comet, they are compressed owing to magnetic field pile-up and draping so that they change in shape. There, the magnetic holes become of comparable size to heavy cometary ion gyroradii, potentially enabling kinetic interactions.
\end{abstract}

Key words. plasmas - magnetohydrodynamics (MHD) - instabilities - comets: general - interplanetary medium

\section{Introduction}

Magnetic holes in the solar wind were defined by Turner et al. (1977) as "distinct depressions, or "holes", in otherwise nearly average conditions", which have a decrease in the interplanetary magnetic field (IMF) strength to below $1 \mathrm{nT}$. The properties of such holes were determined based on data from the Explorer 43 (IMP-6) spacecraft. Turner et al. (1977) gave an occurrence rate of 2.5 day $^{-1}$ and a duration of 2-130 s, which corresponds to a width of around $800-50000 \mathrm{~km}$ or $8-500$ proton gyroradii, assuming a solar wind flow velocity of $400 \mathrm{~km} \mathrm{~s}^{-1}$. The magnetic holes were of two distinct types: first, holes in which the magnetic field depression was collocated with a rotation of the magnetic field and, therefore, with a current sheet, and second, holes without such a rotation, which were called linear holes.

Recent studies of solar wind magnetic holes at $1 \mathrm{AU}$ have confirmed and expanded on these results. Briand et al. (2010), using STEREO data, confirmed that both linear holes and holes associated with IMF directional discontinuities exist, with a relative occurrence rate of 32 and $68 \%$, respectively. In a Cluster study, Xiao et al. (2010) showed that linear solar wind magnetic holes - defined as a minimum decrease of the magnetic field strength of $25 \%$ and a field rotation of less than $10^{\circ}$ across the hole - are likely to have the shape of a rotational ellipsoid elongated along the IMF with a ratio of the field parallel to perpendicular axes radii of 1.93 .

Investigating over 2000 linear magnetic holes observed by Wind, Stevens \& Kasper (2007) showed that there is a balance between magnetic and thermal pressure for a large majority of theses structures at all spatial scales. The authors also investigated the ion temperature anisotropy and found that the deepest holes were found when the solar wind just outside of the holes had a high $\beta$, which is defined as the ratio of thermal to magnetic pressures, and was only marginally stable with respect to the magnetic mirror instability. Finally, Karlsson et al. (2015) investigated so-called diamagnetic plasmoids in the solar wind, using Cluster data. These structures are localized density enhancements correlated with magnetic field decreases and are likely to be magnetic holes in pressure balance. The reported scale sizes of 0.5-30 Earth radii are consistent with the results of Turner et al. (1977).

Solar wind magnetic holes also exist at heliocentric distances other than 1 AU. Winterhalter et al. (1995) used Ulysses data to search for magnetic holes, which are defined as a decrease of the magnetic field strength, $B$, of $50 \%$ relative to a background field strength that is defined by a $300 \mathrm{~s}$ running average. For the whole range of heliocentric distances investigated (1-5.4 AU), both linear holes and holes associated with magnetic field rotations were 
found in large numbers. The heliocentric latitude was within $25^{\circ}$ of the solar equatorial plane all the time. This study was extended by Sperveslage et al. (2000) who used Helios 1, 2, and Voyager 2 data to show that magnetic holes exist close to the ecliptic plane all the way from 0.3 to $17 \mathrm{AU}$. Out of 850 magnetic holes they found $30 \%$ to be linear with less than $10^{\circ}$ change in magnetic field direction according to their definition. Venus Express observations of solar wind magnetic holes at $0.72 \mathrm{AU}$ showed that they have similar properties to holes observed at $1 \mathrm{AU}$. Both linear magnetic holes and holes associated with magnetic field rotations were observed (Zhang et al. 2008b,a, 2009) and at least the linear holes were elongated along the IMF (Zhang et al. 2008a). Finally, magnetic holes have been observed by the MESSENGER spacecraft in the solar wind close to Mercury (at around $0.3-0.4 \mathrm{AU}$ ) and have scale sizes of 400-80000 km (Karlsson et al. 2016).

Solar wind magnetic holes have also been observed away from the ecliptic plane. Fränz et al. (2000) investigated Ulysses data from the south polar pass during 1994, where Ulysses was located below $-45^{\circ}$ solar latitude, and almost continuously embedded in the fast solar wind. They found magnetic holes of durations from a few seconds up to $30 \mathrm{~min}$, which were often bounded by sharp tangential discontinuities. Neugebauer et al. (2001) claimed that such sharp discontinuities are rare in the ecliptic plane, however, they have often been observed by the Cluster spacecraft close to Earth (Spanopoulos 2010; Karlsson et al. 2015).

No consensus on the generation of magnetic holes in the solar wind has been reached. It is possible that linear magnetic holes and holes associated with magnetic field rotations have different origins. Turner et al. (1977) already suggested in their early paper that rotational holes may come about from annihilation of magnetic flux due to slow reconnection in the solar wind (Sweet 1958; Parker 1963). This idea has some support in the fact that the depth of the rotational magnetic holes show a correlation with the amount of magnetic field rotation across the structures (Zurbuchen et al. 2001; Zhang et al. 2008b). An alternative explanation is that these magnetic holes are remnants of structures created at the photosphere. Both Neugebauer et al. (2001) and Zurbuchen et al. (2001) argue against this because of the lack of differences in the chemical composition of magnetic holes and surrounding solar wind.

Linear magnetic holes have been suggested to have an association with magnetic mirror mode waves because the plasma in and close to the holes often is only marginally stable with respect to the mirror mode instability (Winterhalter et al. 1995; Neugebauer et al. 2001; Stevens \& Kasper 2007; Ahmadi et al. 2017). The fact that the magnetic holes are isolated structures, and not part of a periodic chain of structures as one would expect from mirror mode waves, would indicate that linear magnetic holes are remnants of magnetic mirror waves; these linear magnetic holes are possibly the result of coalescing mirror mode structures (Winterhalter et al. 1995; Zhang et al. 2009) and possibly propagating as soliton structures through a medium that is mirror mode stable (Baumgärtel 1999; Sperveslage et al. 2000). Alternative mechanisms include right-handed polarized Alfvén waves, propagating almost perpendicular to the magnetic field in the presence of temperature anisotropies (Buti et al. 2001), interaction of nonlinear Alfvén and fast waves (Vasquez \& Hollweg 1999), and Alfvén wave steepening (Tsurutani et al. 2002).

Interestingly, structures very similar to solar wind magnetic holes have been observed in the magnetosheath of Earth, although they are compressed when they pass the bow shock (Karlsson et al. 2012, 2015). Also, in Mercury's magnetosheath, magnetic holes have been observed to survive the passage of the bow shock (Karlsson et al. 2016). In this context, we note that the term magnetic holes has also been used for trains of periodically occurring decreases of the magnetic field. Such structures have been observed in the magnetosheaths of Saturn (e.g., Cattaneo et al. 1998), Jupiter (e.g., Erdős \& Balogh 1996; Joy et al. 2006), and comet Halley (Russell et al. 1987), but are likely to be magnetic mirror mode waves locally generated owing to the large temperature anisotropies typically found in magnetosheaths (Volwerk et al. 2014). In this paper, we do not consider such structures as magnetic holes.

The fact that magnetic holes can propagate into the magnetosheath indicates that they can affect planetary magnetospheres in different ways. Similar to so-called magnetosheath jets, localized increases of dynamic pressure in the magnetosheath (e.g., Hietala et al. 2009; Plaschke et al. 2013; Karlsson et al. 2015), their excess momentum may result in the generation of surface and compressional waves (Plaschke et al. 2009; Archer et al. 2012; Dmitriev \& Suvorova 2012), generate ionospheric flow enhancements (Hietala et al. 2012), enter the magnetosphere via impulsive penetration (Savin et al. 2012; Gunell et al. 2012; Karlsson et al. 2012), or trigger localized reconnection (Hietala et al. 2018). Furthermore, the low magnetic field values in the holes may affect ion trajectories (Fränz et al. 2000).

The plasma dynamics at a comet (e.g., Glassmeier 2017) are fundamentally different from those at magnetized bodies, such as Earth and Mercury. At a comet, irradiation causes surface and subsurface ices to sublimate and then expand into the surrounding space. These gases are then ionized, mostly by photoionization and electron impact. The newly born ions are essentially at rest in the frame of reference of the comet, but they can be picked up and accelerated to be eventually incorporated into the solar wind flow. This mass loading, in turn, changes the dynamics of the solar wind leading to structures such as the bow shock (Koenders et al. 2013), solar wind ion cavity (Behar et al. 2017), and diamagnetic cavity (Goetz et al. 2016b). For this study, the existence of the solar wind cavity, i.e., the absence or strong reduction of solar wind protons in the inner coma, is of special interest, as we are interested in the propagation of a solar wind structure in the vicinity of the comet. The extent of that cavity (and even its existence) is heavily dependent on the outgassing rate, which increases drastically as the comet approaches the Sun. It was found that the solar wind ion cavity begins to be observed by Rosetta around April 2015. Although the protons are deflected owing to the mass loading, this does not mean that solar wind electrons, which are closely tied to the magnetic field, cannot penetrate the inner coma (Madanian et al. 2017).

Our goal is to investigate if magnetic holes are present in the vicinity of comet 67P/Churyumov-Gerasimenko (67P), which was recently investigated by the Rosetta spacecraft (Glassmeier et al. 2007a). In particular, we are interested in establishing if magnetic holes can be observed in the inner coma of the comet and if they are similar to magnetic holes discovered in the magnetosheath of the Earth. The fact that Rosetta was the first spacecraft to spend an extended time in the inner coma of a comet makes such a study possible. The long time spent by Rosetta escorting the comet also makes it possible to study effects due to variations in outgassing rate.

\section{Data and methods}

The basis of our investigation are magnetic field measurements by the fluxgate Magnetometer (MAG; Glassmeier et al. 2007b) of the Rosetta Plasma Consortium (RPC) instrument suite 
(Carr et al. 2007), taken in April and May 2015 in the vicinity of comet 67P (Glassmeier et al. 2007a). To find candidate magnetic hole events, we adapted the method introduced in Karlsson et al. (2016), which they applied to MESSENGER magnetic field measurements of Mercury's magnetosheath and upstream solar wind. This method is similar to other methods used in prior statistical studies on magnetic holes (e.g., Winterhalter et al. 1995; Sperveslage et al. 2000).

First, we determine background and low-pass magnetic field strength time series $\left(B_{0}\right.$ and $\left.B_{\text {low }}\right)$ by box car averaging over 300 and $10 \mathrm{~s}$, respectively. From the ratio time series $\Delta B / B_{0}=\left(B_{\text {low }}-B_{0}\right) / B_{0}$, lowest depressions are preselected that are separated by at least $300 \mathrm{~s}$. We obtain a set of 11970 decreases in $\Delta B / B_{0}$. From $B_{\text {low }}$ we also calculate the standard deviation $\sigma_{B}$ over a $300 \mathrm{~s}$ running window.

Second, we apply several additional criteria for downselection: (1) $\Delta B / B_{0}$ should reach below $-50 \%$; (2) $B_{0}$ should exceed $15 \mathrm{nT}$; (3) $\sigma_{B}$ should not exceed $5.4 \mathrm{nT}$; and (4) four minutes before and after the event, $\sigma_{B}$ should not exceed half of $\sigma_{B}$ at event time. Criterion 1 was part of the method introduced in Karlsson et al. (2016); it ensures that magnetic holes correspond to significant depressions of the magnetic field. With criterion 2 we seek to keep the relative influence of magnetometer calibration/offset uncertainties (on the order of up to $\sim 5 \mathrm{nT}$ ) on the magnetic field strength low. The threshold value of $15 \mathrm{nT}$ is a trade off between lower relative uncertainties (higher threshold) and a larger number of potential magnetic hole events (lower threshold). Minimizing $\sigma_{B}$ at event time (criterion 3 ) and further before and after the event (criterion 4) enhances the likelihood of selecting clean events that are isolated and not part of a wave train, to be consistent with the usual magnetic hole definitions (e.g., Turner et al. 1977). The threshold level of $5.4 \mathrm{nT}$ (criterion 3 ) is the 20th percentile of the $\sigma_{B}$ distribution pertaining to the times of the preselected events. Applying these criteria, the number of events is reduced to 23 . These events are listed in Table A.1. From those, six example events that are representative of the diversity among the event sample are shown in Fig. 1.

The durations of the magnetic hole events were determined by taking the full width at half minimum of the $\Delta B / B_{0}$ depressions. The solar wind conditions present during the events were determined by Enlil background solar wind runs (Odstrcil et al. 2004) for Carrington Rotations CR 2162/2163 for April and May 2015 at the Community Coordinated Modeling Center (CCMC; https://ccmc.gsfc.nasa.gov/). Enlil/WSA 2.8 was run for the inner heliosphere with the magnetograms provided by Kitt Peak observatory and $2 \mathrm{AU}$ as the outer boundary. The results from the two model runs are shown in Fig. 2, and the solar wind velocities $V_{\mathrm{sw}}$ and magnetic field strengths $B_{\mathrm{sw}}$ estimated for the time of the events at the position of the comet are given in Table A.1.

The Enlil $B_{\mathrm{sw}}$ predictions have been found to be lower by a factor of 2, on average, with respect to in situ observed total magnetic fields, whereas the average model $V_{\text {sw }}$ may differ only by $20 \mathrm{~km} \mathrm{~s}^{-1}$ from the in situ observations. The arrival times of corotating interaction regions should match the observed in situ arrivals to within about one day (for details see Gressl et al. 2014).

For each of the 23 events, we checked whether the RPC Langmuir Probe (LAP) current data are available. The LAP instrument (Eriksson et al. 2007) used two spherical sensors mounted on booms protruding from the spacecraft body. In the current collecting mode, a bias voltage was applied to the spheres, and the current flowing from the sphere to the surrounding plasma was measured. This current is carried by various plasma populations, photoelectrons, and secondary electrons. In this study, we use the continuously sampled probe currents, obtained at a $58 \mathrm{~Hz}$ sampling frequency. Variations in the probe current are mainly due to changes in plasma density and temperature. Often the temperature is assumed constant (see below) and the probe current can then be used to estimate the relative change in plasma density. Table A.1 and Fig. 1 are based on the current from LAP probe 1, which was biased with a positive potential.

Finally, we checked Rosetta Ion and Electron Sensor (IES) data (Burch et al. 2007) for signatures of solar wind ions at the days that the events took place. We found signatures for the days of all events 1-13, until 19 April, and no clear signatures of solar wind ions on the days of events 14-23, from 21 April on. A central horizontal line divides the event groups in Table A.1. This agrees with measurements by the Rosetta Ion Composition Analyser (ICA; Nilsson et al. 2007), which observed clear solar wind proton signatures in April 2015, but only low fluxes of those protons at the end of May 2015, after a larger data gap (Nilsson et al. 2017). We also checked that the detected events are not falsely identified diamagnetic cavity encounters (see Goetz et al. 2016a). This is accomplished by inspecting the electron energy distributions measured by IES. Characteristic particle signatures present near the diamagnetic cavity (Nemeth et al. 2016) could not be found during any of the events listed here.

\section{Results}

Out of the selected 23 events, 14 showed unipolar magnetic field signatures (see Figs. 1a, d, and e), i.e., a decrease followed by an increase in magnetic field strength up to the initial level, whereas nine events showed bipolar signatures of a magnetic field decrease followed by an increase above the initial level (see Figs. 1b, c, and f). Based on figures from past publications on magnetic holes, they have usually been associated with unipolar signatures (e.g., Turner et al. 1977; Winterhalter et al. 1994; Sperveslage et al. 2000; Stevens \& Kasper 2007). Our events show that uni- or bipolarity and the presence of a co-located current sheet seem to be rather independent from each other: 3 out of the 14 unipolar (see Fig. 1e) and 3 out of the 9 bipolar events (see Fig. 1b) feature such current sheets.

The LAP data are available for at least two events per unipolar/bipolar with/without current sheet category. There is almost always, i.e., 14 out of 15 events with LAP data, a clear corresponding LAP current response (suggesting a local density increase) associated with the respective magnetic holes; the exception to this is event number 7. However, there is a shift in the density increase in 7 out of those 14 times (see, e.g., Volwerk et al. 2017); the density responses are then not strictly anticorrelated with the magnetic field depressions (Figs. 1a and b), but are measured slightly but noticeably after those depressions (Figs. 1c-f). Clear anti-correlations are found to be associated with unipolar magnetic holes without current sheets and bipolar magnetic holes with current sheets. This is apparent in Table 1, which shows to which categories the 14 events with clear density signatures belong. Furthermore, it can be seen that all the cases of clear anti-correlation are associated with the clear presence of solar wind ions in April 2015. All May 2015 events, instead, show a shift between magnetic field and density and no clear signatures of solar wind ions. It should be noted that the six events shown in Fig. 1 are representatives of all the categories populated in Table 1.

The behavior of anti-correlation/shift between magnetic field and density data can also be related to the distance of Rosetta 
April 2015 - more solar wind ions

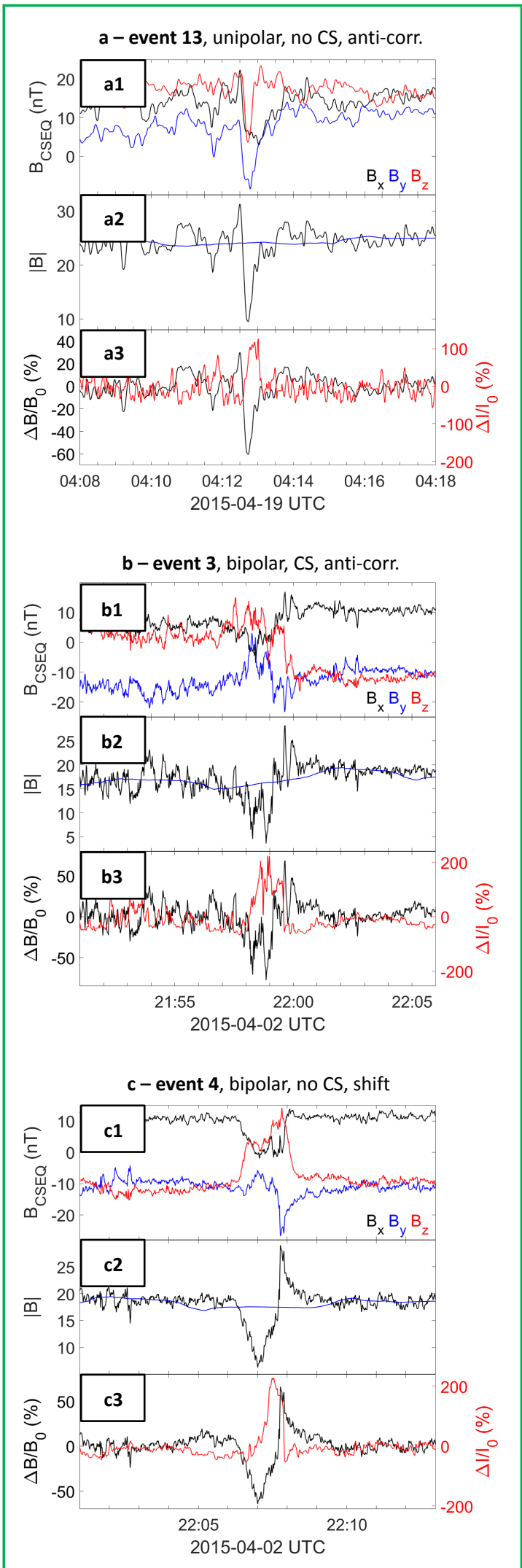

May 2015 - less solar wind ions

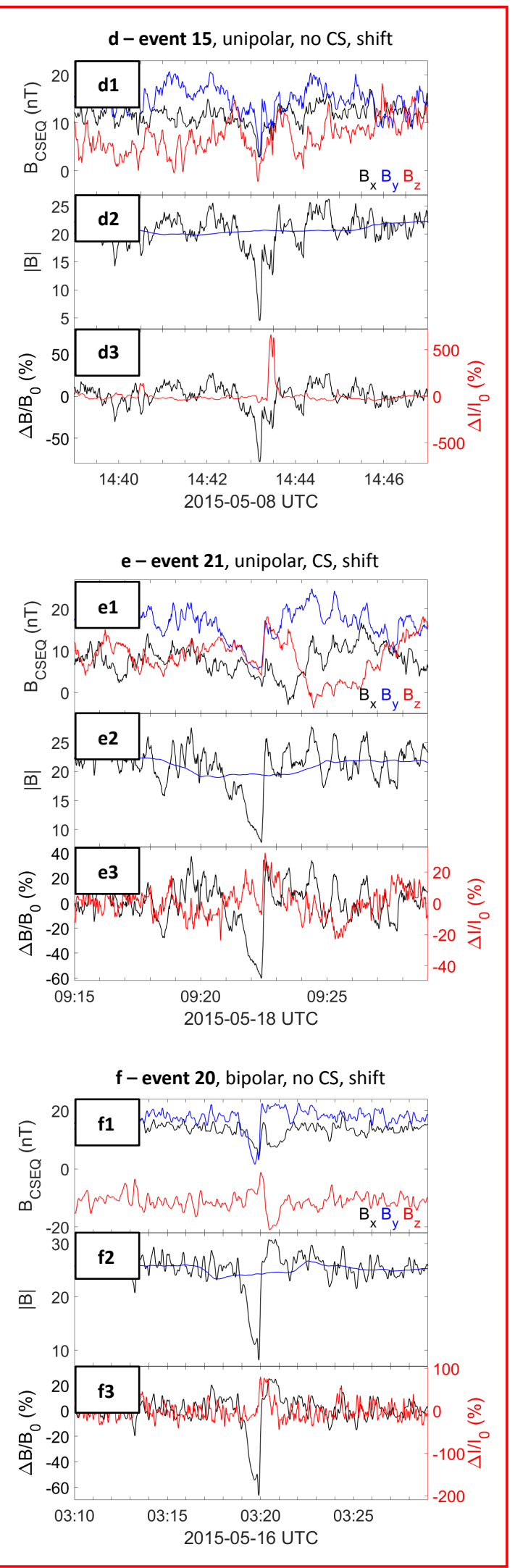

Fig. 1. Six example events: numbers (a) 13, (b) 3, (c) 4, (d) 15 , (e) 21 , and (f) 20 . Panels $a-c$ and $d-f$ : pertain to events with clear/unclear presence of solar wind ions from April and May 2015, respectively. Subpanels: (1) magnetic field components in cometocentric solar equatorial (CSEQ) coordinates, (2) $B_{\text {low }}$ in black and $B_{0}$ in blue, and (3) $\Delta B / B_{0}$ in black and relative change in LAP current $\Delta I / I_{0}$ in red. 

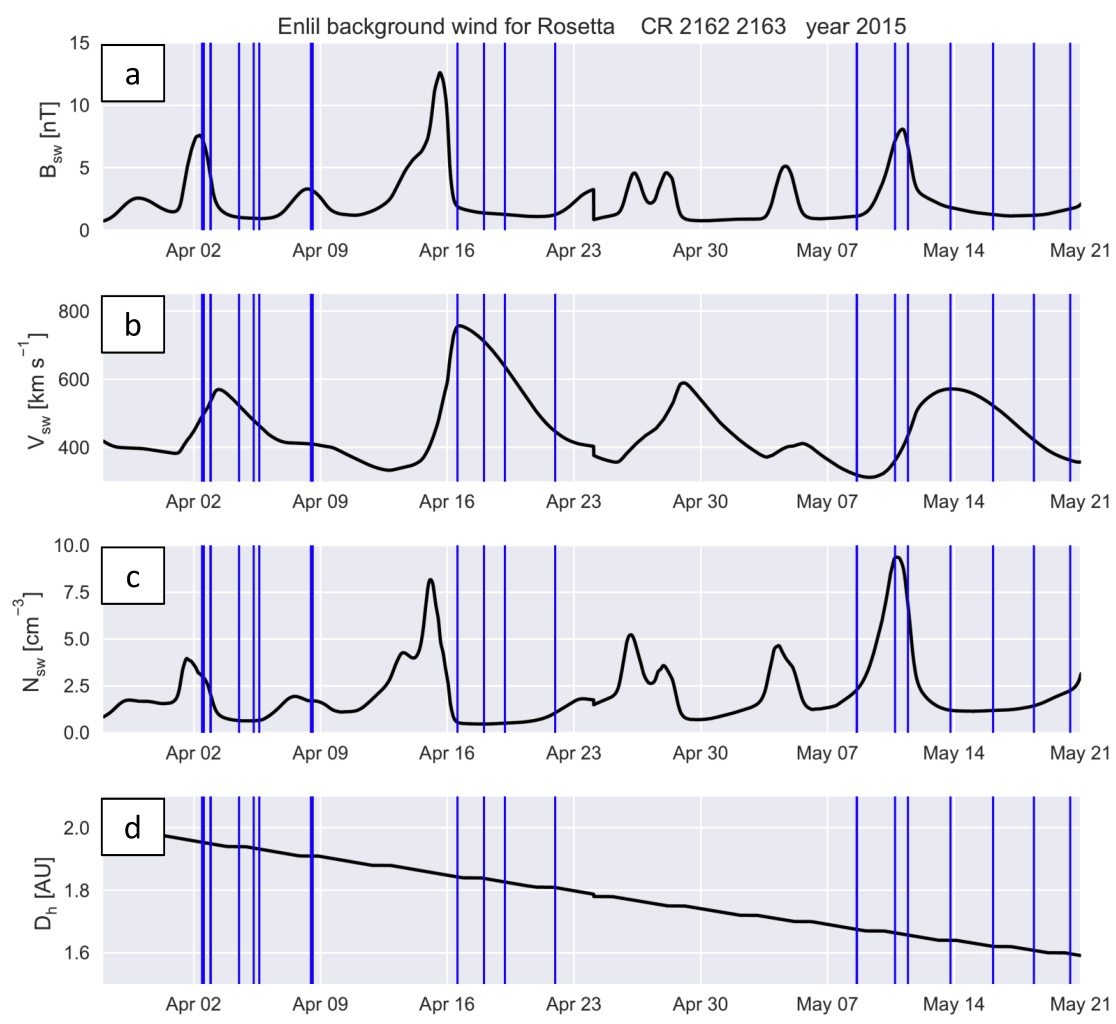

Fig. 2. Enlil model-derived solar wind conditions for the position of comet 67P in April and May 2015. From top to bottom panels: (a) IMF strength $B_{\mathrm{sw}}$, (b) solar wind velocity $V_{\mathrm{sw}},(c)$ solar wind density $N_{\mathrm{sw}}$, and $(d)$ distance $D_{\mathrm{h}}$ to the Sun. Magnetic hole events are indicated by blue vertical lines.

Table 1. Categories of the 14 events with clear LAP peaks associated with the magnetic holes.

\begin{tabular}{ccc}
\hline \hline Category & Anti-correlation & Shift \\
\hline unipolar with CS & & $\mathrm{N}_{16} \mathrm{~N}_{21}$ \\
unipolar without CS & $\mathrm{S}_{1} \mathrm{~S}_{2} \mathrm{~S}_{8} \mathrm{~S}_{10} \mathrm{~S}_{13}$ & $\mathrm{~N}_{15}$ \\
bipolar with CS & $\mathrm{S}_{3} \mathrm{~S}_{12}$ & \\
bipolar without CS & & $\mathrm{S}_{4} \mathrm{~S}_{5} \mathrm{~S}_{9} \quad \mathrm{~N}_{20}$ \\
\hline
\end{tabular}

Notes. The values $\mathrm{S}$ and $\mathrm{N}$ denote events with $(\mathrm{S})$ and without $(\mathrm{N})$ the clear presence of solar wind ions, observed in April (S) and May $(\mathrm{N})$, respectively. The subscripts are the event numbers as given in Table A.1.

from the comet $\left(D_{\mathrm{c}}\right)$ and the distance of the comet from the Sun $\left(D_{\mathrm{h}}\right)$ because of the changing cometary activity (Hansen et al. 2016), as shown in Table A.1. From the beginning to the end of April, Rosetta gradually approached the comet; it then stayed relatively close to the comet during May. In addition, over the two months considered, the comet and Rosetta were on the inbound trajectory toward the Sun. Hence, their heliocentric distance $D_{\mathrm{h}}$ continuously decreased from about 2 to $1.5 \mathrm{AU}$. Figure 3 shows the occurrence of all 23 magnetic hole events as a function of $D_{\mathrm{c}}$ (a) and $D_{\mathrm{h}}$ (b). As can be seen in the bottom panels, Fig. $3 \mathrm{a} 3$ and b3, the relative/normalized occurrence is larger at larger distances from the comet and Sun, both corresponding to earlier times in the April to May 2015 frame.

Finally, the duration of the magnetic holes (see Table A.1) does not seem to be associated with any of the other features/occurrences mentioned above. The maximum and minimum dwell times of Rosetta within the magnetic holes are 175.8 and 23.9 s, corresponding to events 3 and 18, respectively. By taking into account the Enlil model estimated solar wind velocities $V_{\text {sw }}$ (see Table A.1 and Fig. 2b), we can transform those numbers into scale size estimates of the magnetic holes in the solar wind, upstream of the cometary environment. We obtain a median value of $21.9 \times 10^{3} \mathrm{~km}$ (maximum and minimum values are $93.9 \times 10^{3}$ and $10.4 \times 10^{3} \mathrm{~km}$, respectively). We note that these scale sizes correspond to the upstream, pristine solar wind environment. Near the comet, however, the magnetic holes should be compressed by the ratio of the local magnetic field strength $B_{0}$ and the upstream IMF values $B_{\mathrm{sw}}$; both values are indicated in Table A.1 for all the events. The $B_{0} / B_{\mathrm{sw}}$ compression ratios range between 2.1 and 20.4 , higher compression ratios tending to be more common for later events in the April-May 2015 time frame. We obtain scale sizes of the magnetic holes at the comet along the comet-Sun-line between $0.7 \times 10^{3}$ and $24.8 \times 10^{3} \mathrm{~km}$. The median of these values for all 23 events is $2.2 \times 10^{3} \mathrm{~km}$, i.e., almost exactly one order of magnitude lower than in the solar wind. From the scale sizes and durations of magnetic holes, we can also deduce the velocities $V_{\mathrm{c}}=B_{\mathrm{sw}} V_{\mathrm{sw}} / B_{0}$ of the magnetic structures in the vicinity of the comet. These are shown in Table A.1, as well. As can be seen, they range from 16 to $232 \mathrm{~km} \mathrm{~s}^{-1}$; the median velocity is $40 \mathrm{~km} \mathrm{~s}^{-1}$. These velocities should correspond to the convection speeds of the plasma near the comet. They are in excellent agreement with RPC-ICA observations of partially picked up water ions, moving anti-sunward at some tens of kilometer per second (see Fig. 4 of Berčič et al. 2018).

We note that the Enlil estimates of the magnetic field strength typically underestimate in situ observations of the IMF strength, on average by a factor of 2 as found by Gressl et al. (2014). Taking this into account, we would obtain lower compression ratios $B_{0} /\left(2 B_{\text {sw }}\right)$ between 1.1 and 10.2 , and larger scale size estimates of the magnetic holes at the comet, between $1.3 \times 10^{3} \mathrm{~km}$ and $49.5 \times 10^{3} \mathrm{~km}$. In this case, the corrected median scale size would be: $4.0 \times 10^{3} \mathrm{~km}$. Also, the velocities $V_{\mathrm{c}}$ of the magnetic structures near the comet would be larger by a factor of 2 , yielding a median velocity of $80 \mathrm{~km} \mathrm{~s}^{-1}$. 

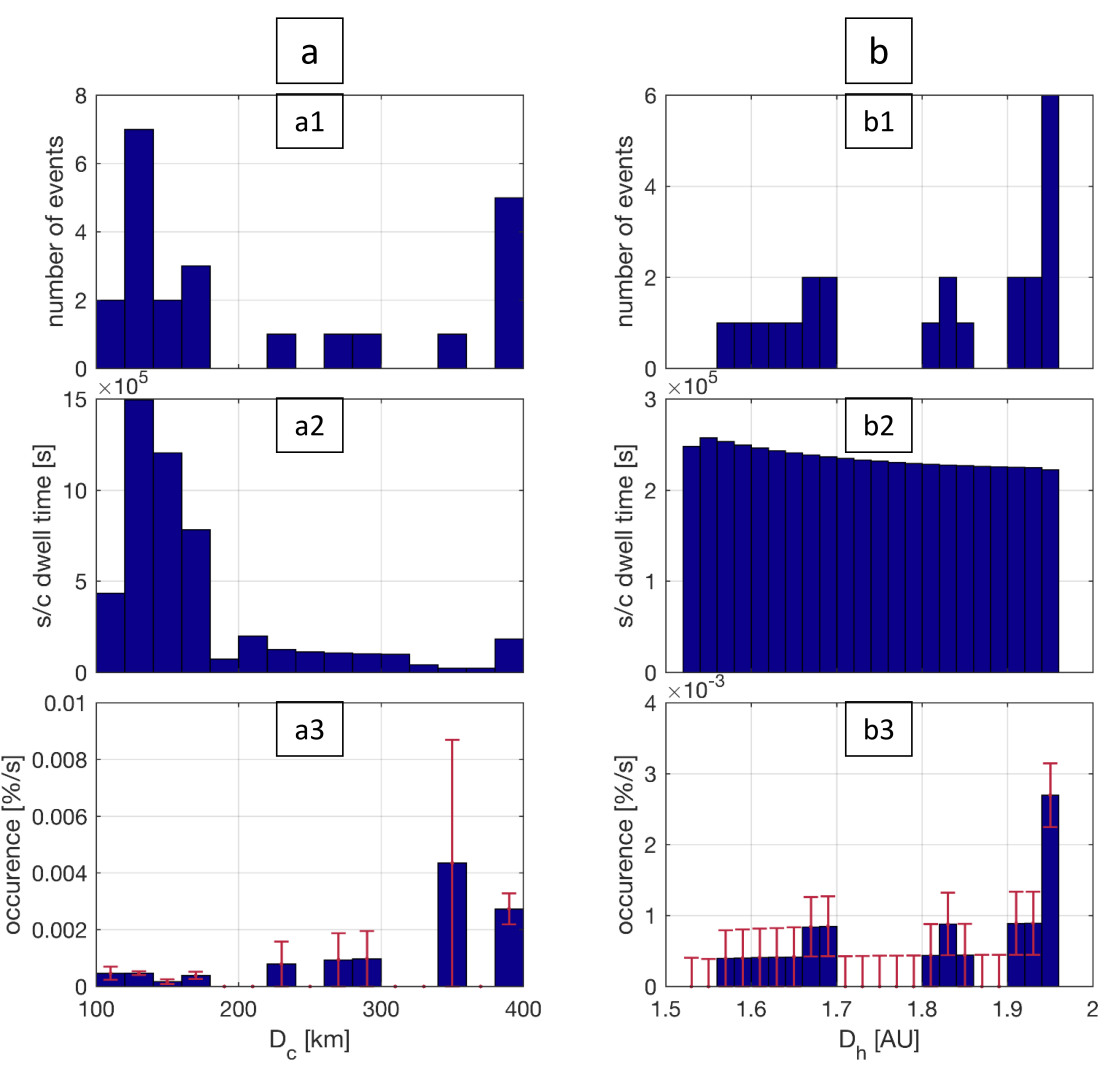

Fig. 3. Occurrence of events as a function of distance to the comet $D_{\mathrm{c}}(a)$ and distance to the Sun $D_{\mathrm{h}}(b)$. From top to bottom panels: show number of events (1), dwell time of Rosetta (2), and normalized occurrence of events (3). Error bars show the one count errors.

\section{Discussion}

Rosetta enables us to show, for the first time, that magnetic holes are regularly observable inside the coma of a comet, at heliocentric distances between 1.5 and $2 \mathrm{AU}$. The events have some properties that are similar to earlier observations of magnetic holes. Their timescales are on the order of one minute, as seen also by Turner et al. (1977) or Karlsson et al. (2015), for instance. They are associated with density increases, which is also a wellknown feature (Stevens \& Kasper 2007; Karlsson et al. 2015). Furthermore, as known from prior observations, we see two different types of magnetic holes: linear holes and magnetic holes associated with current sheets.

It is remarkable that such structures even survive when solar wind ions are mostly replaced by heavy cometary ions in May 2015. The solar wind electrons should play an important role in the conservation and propagation of IMF structures, such as magnetic holes, through the coma (Madanian et al. 2017; Deca et al. 2017). However, in May 2015, the magnetic holes actually look slightly different from the April events, all featuring shifts of the density enhancements toward their trailing edges. Interestingly, this behavior can also be observed near Earth. A reexamination of the magnetic holes reported by Karlsson et al. (2015), observed by Cluster, shows that their structure is more complicated in the magnetosheath of the Earth than in the solar wind, where magnetic holes are typically unipolar with wellcorrelated density increases: downstream of the bow shock of the Earth, magnetic holes are also sometimes associated with (1) bipolar signatures, (2) shifts in the density, and (3) a generally lower rate of occurrence. This behavior can be seen in Fig. 4, which shows a typical solar wind and a typical magnetosheath magnetic hole case (panels a and $b$, respectively). The magnetosheath event also displays a shifted density peak and an additional temperature maximum inside the magnetic hole, which perhaps helps maintain the pressure balance. This picture emerging from the Cluster observations (points 1,2, and 3) is in agreement with our observations:

(1) In April and May, Rosetta does not observe pristine solar wind (Nilsson et al. 2017), and therefore the magnetic holes are processed structures either because they already passed through a cometary bow shock, if it existed at that time, and/or because of the mass loading process of the solar wind. This situation is more comparable to the situation encountered by Cluster inside the magnetosheath. Similarly, we see about half of the magnetic holes featuring bipolar structures (Figs. 1b, c, f, and 4b) instead of unipolar magnetic field variations.

(2) In fact, as time progresses from April to May 2015, Rosetta finds itself deeper inside the cometosheath, as it moves closer to the nucleus (lower $D_{\mathrm{c}}$ ), and as the comet moves toward the Sun (lower $D_{\mathrm{h}}$ ) so that the cometary activity (out-gassing) picks up: the gas production rate increases from $0.8 \times 10^{27}$ to $3 \times 10^{27} \mathrm{~s}^{-1}$ (Hansen et al. 2016). Consequently, magnetic holes observed in May in the near absence of solar wind ions should be more processed than those seen in April, when solar wind ions were more abundant. This is in agreement with magnetic field/density anti-correlation being the norm in April, whereas in May the density always appears to be shifted with respect to the magnetic field depressions. At this point, we lack an explanation for what could cause that shift in density. We may, however, point out, that the mass loading at the comet does not seem to be the reason, as the same effect is seen at Earth. What could be common to both environments is the presence of a bow shock. Burgess \& Schwartz (1988), for instance, showed the result of a low magnetic field region passing through a bow shock. The magnetic hole is conserved while passing into the magnetosheath, but it is modified 

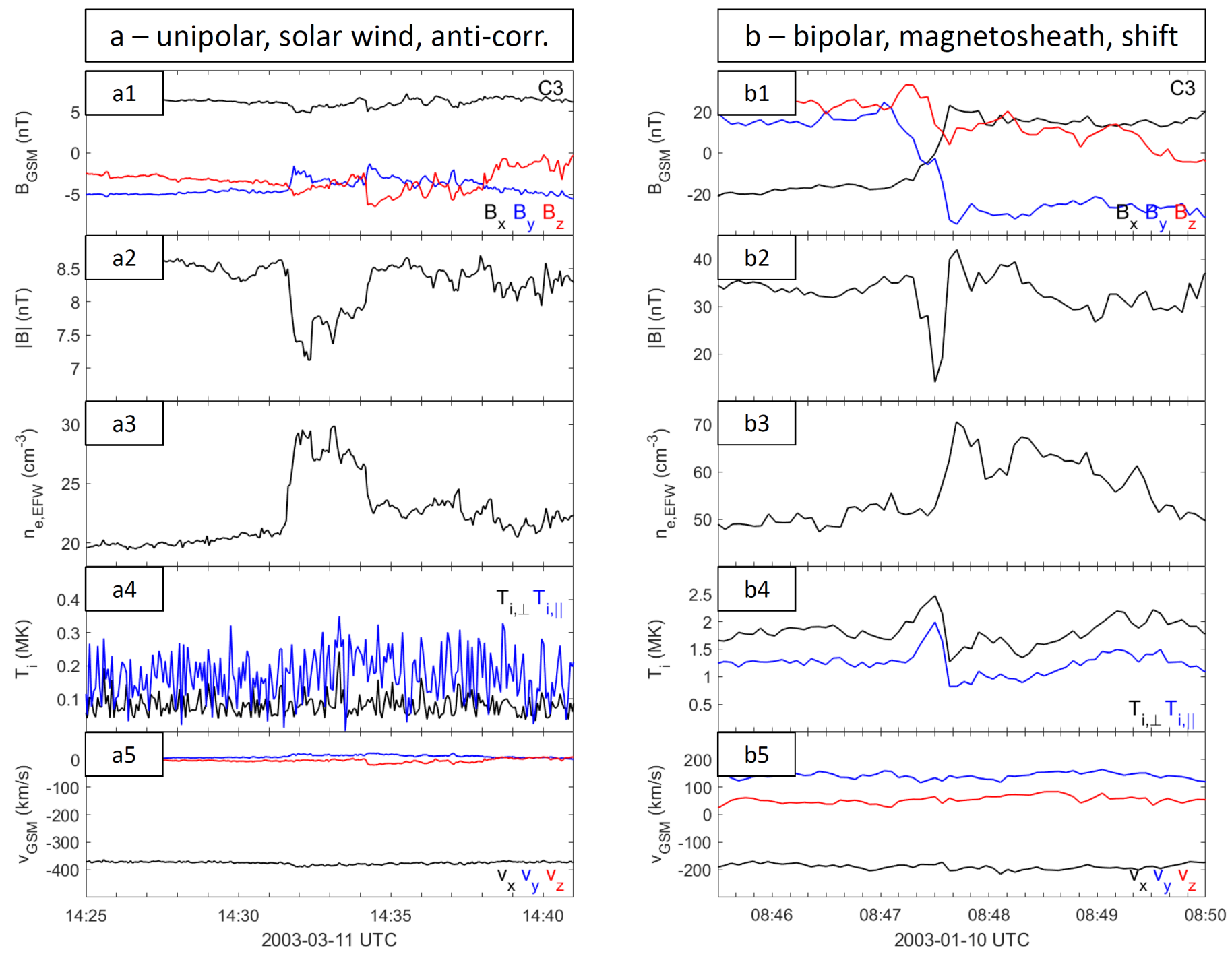

Fig. 4. Two magnetic hole examples from Karlsson et al. (2015). Left panel: cluster 3 interval from 11 March 2003, showing a unipolar magnetic hole in the solar wind with anti-correlated density variation. Right panel: cluster 3 interval from 10 January 2003, showing a bipolar magnetic hole in the terrestrial magnetosheath, co-located with a current sheet and featuring a shifted density response. From top to bottom panels: (1) magnetic field in geocentric solar magnetospheric (GSM) coordinates, (2) modulus of the magnetic field, (3) electron density derived from the Electric Fields and Waves (EFW) instrument, (4) ion temperatures in magnetic field parallel (blue) and perpendicular (black) directions, and (5) ion velocity in GSM.

into a more complicated structure. At the comet, the presence of a bow shock is predicted for the April-May 2015 time frame (Koenders et al. 2013). However, Rosetta never actually crossed a bow shock in front of comet $67 \mathrm{P}$, and it is unclear whether a bow shock actually developed around perihelion. Hence, it would be intriguing if shifts in density with respect to the magnetic field depressions could be linked to magnetic holes passing a bow shock, as those shifts would then be a marker for the upstream presence of a bow shock.

It should be noted that we implicitly assume here the LAP current variations (panels 3 in Fig. 1) to be proportional to the plasma density variations. This is, however, only true when constant temperature and spacecraft potential can be assumed. As we see in the Cluster measurements (Fig. 4b4), this may not be fulfilled. In this case,

$\frac{\Delta I}{I_{0}}=\frac{\Delta N}{N_{0}}+\frac{\Delta T}{2 T_{0}}$ holds (Eriksson \& Boström 1995). However, we can argue that the current variations are indeed due to density variations: for the interval investigated here, the two probes of the LAP instrument are kept at potentials of different signs (positive/negative), hence, attracting electrons/ions, respectively. As a consequence of quasi-neutrality, the ion and electron charge densities are the same, while the temperatures of both species usually behave differently. Hence, correlated probe currents indicate density variations, while uncorrelated current variations would indicate temperature variations. We have inspected LAP probe 2 data for all events, for which LAP data are available. For all of those, except event number 7 (unclear LAP signature), we found the currents from probes 1 and 2 to be highly correlated. In addition, the ion current should depend on the ion composition. However, if the composition is constant, the change in the ion current is still proportional to the density change. In any case, during this part of the mission the density of $\mathrm{H}_{2} \mathrm{O}^{+}$is much larger than the solar wind proton density (Nilsson et al. 2017) and 
any effect of the latter is negligible. We can also note that photoelectrons from the spacecraft body do not affect the measurements, since the presence of a warm, dense electron population during this part of the mission drives the spacecraft to a negative potential on the order of a few tens of $\mathrm{V}$ (Odelstad et al. 2017). This expels the photoelectrons from the vicinity of the spacecraft. Hence, we conclude that the LAP current variations are due to density variations of the cometary plasma.

(3) Furthermore, the magnetic hole occurrence increases with distance $D_{\mathrm{c}}$ to the comet and also with distance $D_{\mathrm{h}}$ to the Sun (see Fig. 3). Both increased $D_{\mathrm{c}}$ and $D_{\mathrm{h}}$ correspond to Rosetta being less immersed into the coma. Cluster findings of lower magnetic hole occurrence rates in the magnetosheath with respect to the pristine solar wind are in agreement with this. However, as the cometary activity grows when approaching perihelion, the increase in compressional magnetic field fluctuations in the vicinity of the comet in any case makes the identification of isolated magnetic holes impossible, even if they were to make it into the inner coma.

At this point, it should be noted that the magnetic hole events are clustered around fast solar wind streams, as can be seen in Fig. 2b. This agrees with prior observations of magnetic holes being more common around interactions regions of solar wind plasmas of different velocities (Winterhalter et al. 1994, 2000). There, plasma volumes of larger ion beta than typical for the solar wind are known to exist, and are prone to be mirror mode unstable. The mirror mode instability is a candidate mechanism for the formation of magnetic holes, as described in the introduction.

In principle, Rosetta should be able to observe magnetic holes in the solar wind, at larger heliocentric distances. However, the IMF strength (on the order of $1 \mathrm{nT}$ ) is then low in comparison to the uncertainties in the offsets of the magnetic field measurements (on the order of $5 \mathrm{nT}$; see, Goetz et al. 2016a, 2017). The incorrect choice of those offsets completely changes the signatures of the magnetic holes in the field observations, again making their identification impossible. During April and May 2015, the conditions were optimal for observing the magnetic holes with Rosetta; cometary activity was sufficiently high to pile up the IMF above the offset uncertainty levels, but not quite so high as to swamp the magnetic field observations with variations of cometary origin. This justifies, a posteriori, our choice of April and May 2015 measurements for this study.

Finally, we discuss scale sizes of the magnetic holes. We find $2 \times 10^{4} \mathrm{~km}$ to be a typical size of the magnetic holes in the upstream, pristine solar wind. This size is comparable to what has been previously reported (Turner et al. 1977; Fränz et al. 2000; Karlsson et al. 2012, 2015). This size would be larger than the cometary interaction region with the solar wind, for example, when measured by the distance of a possible bow shock from the cometary nucleus (Koenders et al. 2013). In that sense, we could imagine a magnetic hole passing over the comet as a rapid succession of different solar wind conditions. The comet and its environment would essentially fall entirely into the magnetic hole.

This picture is, however, too simple. On the one hand, the size of typical magnetic holes in the solar wind is comparable to the gyroradii of the heavy cometary pick-up ions (e.g., Koenders et al. 2013; Behar et al. 2016). On the other hand, the IMF is piled up at the comet owing to solar wind mass loading, and this process should also drastically reduce the size of the hole there along the comet-Sun line. Taking into account a systematic underestimation of the Enlil predicted IMF strengths $B_{\text {sw }}$ by a
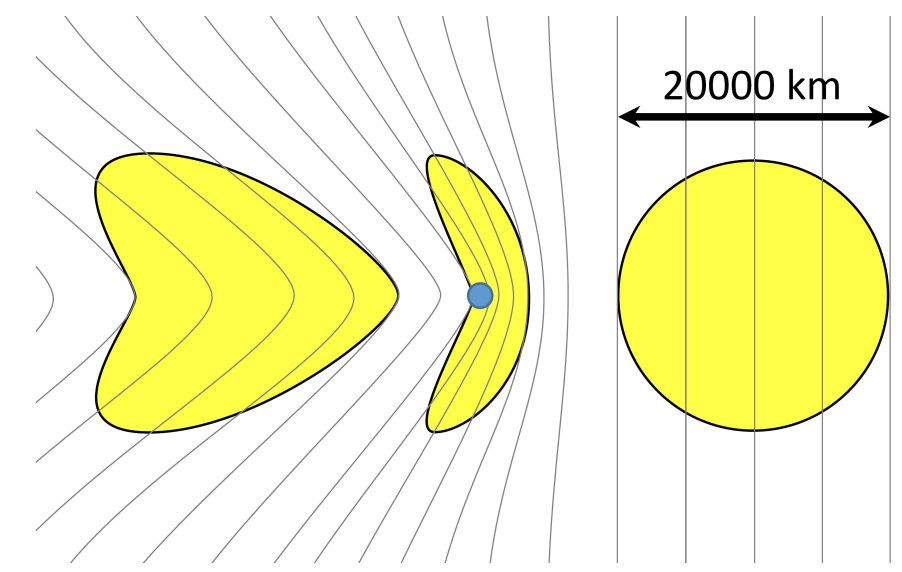

Fig. 5. Sketch of deformation of a magnetic hole (yellow) due to IMF pile up and draping at the cometary nucleus (position in blue); solar wind and magnetic hole are approaching the comet from the right. Field configuration (gray) adapted from Fig. 10d of Simon Wedlund et al. (2017). Far away from the comet adjacent field lines are $5000 \mathrm{~km}$ apart.

factor of 2 (Gressl et al. 2014), we find the magnetic field compression ratio to be on the order of 5 . Hence, typical magnetic hole scale sizes in the cometary environment are on the order of $4 \times 10^{3} \mathrm{~km}$. Although this scale should be regarded as a lower limit because the magnetic field pile up is most pronounced right at the comet where Rosetta is located, this suggests that magnetic holes are of comparable size (at the comet) to the cometary interaction region (see Fig. 5). Hence, we expect the presence of a magnetic hole to influence the gyromotion of at least the heavy cometary ions (Behar et al. 2017). Magnetic holes are large structures at the comet and are kinetic with respect to the heavy cometary ions. This contrasts with the influence that magnetic holes have on the terrestrial plasma environment. Near Earth, the holes are much larger than the solar wind proton gyroradii, but smaller than the system size of bow shock and magnetosphere. At Earth, magnetic holes are small scale, yet entirely magnetohydrodynamic structures. The situation near the comet seems to be more similar to the situation at Mercury rather than at the Earth (Karlsson et al. 2016).

The compression of the magnetic hole structure at the comet is also associated with a slowdown of the corresponding section of the structure. We note that the draping in the immediate vicinity of the nucleus, at distances $D_{\mathrm{c}}$ on the order of $100 \mathrm{~km}$ or below, is expected to be significantly more influenced by kinetic effects than what is shown in Fig. 5 (Koenders et al. 2016). Further away from the comet, at distances $D_{\mathrm{c}}$ on the order of $10^{4} \mathrm{~km}$, however, that structure should be convected with the solar wind plasma at higher velocities. Thus, a spherical magnetic hole should change its shape into a bowl-like structure in 3D. After passing the comet, the magnetic tension of the draped IMF should quickly act in restoring the original shape of the hole (see, again, Fig. 5). It should be noted, however, that some asymmetries in the IMF draping are still expected to persist at larger distances from the comet (Koenders et al. 2015), which are not illustrated in Fig. 5. Also, the kinetic interaction of a magnetic hole with the heavy cometary ions is neglected in the figure. Future simulations on this interaction should show these differences in detail.

\section{Conclusions}

In this study, we show for the first time that magnetic hole structures exist in a cometary environment. Based on this 
fundamental observation, we investigated their properties and reach the following main conclusions:

1. Magnetic holes have many properties in common with previously studied solar wind magnetic holes: density enhancements, scale sizes $\left(1-9 \times 10^{4} \mathrm{~km}\right)$, association or not to current sheets, and association with fast solar wind.

2. Magnetic holes appear to be processed, bearing some similarities with magnetic holes in the terrestrial magnetosheath: shift in density increase with respect to magnetic field minimum and bipolar signatures in the magnetic field strength. The occurrence of shifted density signatures is more pronounced in May than in April, corresponding with lower distances of Rosetta to the nucleus and increased cometary activity levels.

3. Although solar wind protons are mostly replaced by cometary ions in May, magnetic holes are still observed in the inner coma.

4. Magnetic holes are compressed in the interaction with the cometary plasma and should have a significant repercussion onto that plasma, as the compressed scale sizes become similar to the heavy ion gyroradius.

The exact interactions of such magnetic hole structures with all the plasma populations around a comet are still to be investigated, requiring detailed hybrid or particle-in-cell simulations.

Acknowledgements. Without the tremendous work of the Rosetta Science Ground Segment (RSGS), Rosetta Mission Operation Control (RMOC), and all instrument team planners, this study would have been impossible. The Rosetta Plasma Consortium Magnetometer (MAG) and Langmuir Probe (LAP) data are available through the Planetary Science Archive (PSA) of the European Space Agency (ESA) and the Planetary Data System (PDS) of the National Aeronautics and Space Administration (NASA). Rosetta is an ESA mission with contributions from its member states and from NASA. The work on MAG was financially supported by the German Ministerium für Wirtschaft und Energie and the Deutsches Zentrum für Luft- und Raumfahrt under contract 50QP 1401. We acknowledge $\mathrm{H}$. Nilsson for the use of ICA data and J. Burch for the use of IES data. Solar wind simulation results have been provided by the Community Coordinated Modeling Center at Goddard Space Flight Center through their public "Runs on Request" system (http://ccmc.gsfc.nasa.gov). The Enlil Model was developed by D. Odstrcil at the University of Colorado in Boulder. C.M. thanks the Austrian Science Fund (FWF): [P26174-N27] for support.

\section{References}

Ahmadi, N., Germaschewski, K., \& Raeder, J. 2017, Phys. Plasmas, 24, 122121 Archer, M. O., Horbury, T. S., \& Eastwood, J. P. 2012, J. Geophys. Res., 117, A 05228

Baumgärtel, K. 1999, J. Geophys. Res., 104, 28295

Behar, E., Lindkvist, J., Nilsson, H., et al. 2016, A\&A, 596, A42

Behar, E., Nilsson, H., Alho, M., Goetz, C., \& Tsurutani, B. 2017, MNRAS, 469, S396

Berčič, L., Behar, E., Nilsson, H., et al. 2018, A\&A, 613, A57

Briand, C., Soucek, J., Henri, P., \& Mangeney, A. 2010, J. Geophys. Res., 115, A12113

Burch, J. L., Goldstein, R., Cravens, T. E., et al. 2007, Space Sci. Rev., 128, 697

Burgess, D., \& Schwartz, S. J. 1988, J. Geophys. Res., 93, 11327

Buti, B., Tsurutani, B., Neugebauer, M., \& Goldstein, B. 2001, Geophys. Res. Lett., 28, 1355

Carr, C., Cupido, E., Lee, C. G. Y., et al. 2007, Space Sci. Rev., 128, 629

Cattaneo, M. B. B., Basile, C., Moreno, G., \& Richardson, J. D. 1998, J. Geophys. Res., 103, 11961

Deca, J., Divin, A., Henri, P., et al. 2017, Phys. Rev. Lett., 118, 205101

Dmitriev, A. V., \& Suvorova, A. V. 2012, J. Geophys. Res., 117, A08217

Erdős, G., \& Balogh, A. 1996, J. Geophys. Res., 101, 1
Eriksson, A. I., \& Boström, R. 1995, Measurements of Plasma Density Fluctuations and Electric Wave Fields Using Spherical Electrostatic Probes (Kiruna: IRF Swedish Institute of Space Physics), Tech. Rep., 220

Eriksson, A. I., Boström, R., Gill, R., et al. 2007, Space Sci. Rev., 128, 729

Fränz, M., Burgess, D., \& Horbury, T. 2000, J. Geophys. Res., 105, 12725

Glassmeier, K.-H. 2017, Philos. Trans. R. Soc. London, 375, 20160256

Glassmeier, K.-H., Boehnhardt, H., Koschny, D., Kührt, E., \& Richter, I. 2007a, Space Sci. Rev., 128, 1

Glassmeier, K.-H., Richter, I., Diedrich, A., et al. 2007b, Space Sci. Rev., 128, 649

Goetz, C., Koenders, C., Hansen, K. C., et al. 2016a, MNRAS, 462, S459

Goetz, C., Koenders, C., Richter, I., et al. 2016b, A\&A, 588, A24

Goetz, C., Volwerk, M., Richter, I., \& Glassmeier, K.-H. 2017, MNRAS, 469, $\mathrm{S} 268$

Gressl, C., Veronig, A. M., Temmer, M., et al. 2014, Sol. Phys., 289, 1783

Gunell, H., Nilsson, H., Stenberg, G., et al. 2012, Phys. Plasmas, 19, 072906

Hansen, K. C., Altwegg, K., Berthelier, J.-J., et al. 2016, MNRAS, 462, S491

Hietala, H., Laitinen, T. V., Andréeová, K., et al. 2009, Phys. Rev. Lett., 103, 245001

Hietala, H., Partamies, N., Laitinen, T. V., et al. 2012, Ann. Geophys., 30, 33

Hietala, H., Phan, T. D., Angelopoulos, V., et al. 2018, Geophys. Res. Lett., 45, 1732

Joy, S., Kivelson, M., Walker, R., et al. 2006, J. Geophys. Res., 111, A12212

Karlsson, T., Brenning, N., Nilsson, H., et al. 2012, J. Geophys. Res., 117, A03227

Karlsson, T., Kullen, A., Liljeblad, E., et al. 2015, J. Geophys. Res., 120, 7390

Karlsson, T., Liljeblad, E., Kullen, A., et al. 2016, Planet. Space Sci., 129, 61

Koenders, C., Glassmeier, K.-H., Richter, I., Motschmann, U., \& Rubin, M. 2013 Planet. Space Sci., 87, 85

Koenders, C., Glassmeier, K.-H., Richter, I., Ranocha, H., \& Motschmann, U. 2015, Planet. Space Sci., 105, 101

Koenders, C., Goetz, C., Richter, I., Motschmann, U., \& Glassmeier, K.-H. 2016, MNRAS, 462, S235

Madanian, H., Cravens, T. E., Burch, J., et al. 2017, ApJ, 153, 30

Nemeth, Z., Burch, J., Goetz, C., et al. 2016, MNRAS, 462, S415

Neugebauer, M., Goldstein, B., Winterhalter, D., et al. 2001, J. Geophys. Res., 106,5635

Nilsson, H., Lundin, R., Lundin, K., et al. 2007, Space Sci. Rev., 128, 671

Nilsson, H., Wieser, G. S., Behar, E., et al. 2017, MNRAS, 469, S252

Odelstad, E., Stenberg-Wieser, G., Wieser, M., et al. 2017, MNRAS, 469, S568

Odstrcil, D., Riley, P., \& Zhao, X. P. 2004, J. Geophys. Res., 109, A02116

Parker, E. N. 1963, ApJS, 8, 177

Plaschke, F., Glassmeier, K.-H., Sibeck, D. G., et al. 2009, Ann. Geophys., 27, 4521

Plaschke, F., Hietala, H., \& Angelopoulos, V. 2013, Ann. Geophys., 31, 1877

Russell, C., Riedler, W., Schwingenschuh, K., \& Yeroshenko, Y. 1987, Geophys. Res. Lett., 14, 644

Savin, S., Amata, E., Zelenyi, L., et al. 2012, Ann. Geophys., 30, 1

Simon Wedlund, C., Alho, M., Gronoff, G., et al. 2017, A\&A, 604, A73

Spanopoulos, G. 2010, Master's Thesis, KTH, Space and Plasma Physics

Sperveslage, K., Neubauer, F., Baumgärtel, K., \& Ness, N. 2000, Nonlinear Proc. Geoph., 7, 191

Stevens, M., \& Kasper, J. 2007, J. Geophys. Res., 112, A05109

Sweet, P. 1958, Proc. IAU Symp., 6, 123

Tsurutani, B., Dasgupta, B., Galvan, C., et al. 2002, Geophys. Res. Lett., 29, 2233

Turner, J., Burlaga, L., Ness, N., \& Lemaire, J. 1977, J. Geophys. Res., 82, 1921

Vasquez, B. J., \& Hollweg, J. V. 1999, J. Geophys. Res., 104, 4681

Volwerk, M., Glassmeier, K.-H., Delva, M., et al. 2014, Ann. Geophys., 32, 1441

Volwerk, M., Jones, G. H., Broiles, T., et al. 2017, J. Geophys. Res., 122, 3308

Winterhalter, D., Neugebauer, M., Goldstein, B. E., et al. 1994, J. Geophys. Res., 99,23

Winterhalter, D., Neugebauer, M., Goldstein, B., et al. 1995, Space Sci. Rev., 72, 201

Winterhalter, D., Smith, E. J., Neugebauer, M., Goldstein, B. E., \& Tsurutani, B. T. 2000, Geophys. Res. Lett., 27, 1615

Xiao, T., Shi, Q. Q., Zhang, T. L., et al. 2010, Ann. Geophys., 28, 1695

Zhang, T., Russell, C., Baumjohann, W., et al. 2008a, Geophys. Res. Lett., 35, L10106

Zhang, T., Russell, C., Zambelli, W., et al. 2008b, Geophys. Res. Lett., 35, L24102

Zhang, T., Baumjohann, W., Russell, C., et al. 2009, J. Geophys. Res., 114, A10107

Zurbuchen, T., Hefti, S., Fisk, L., et al. 2001, J. Geophys. Res., 106, 16001 


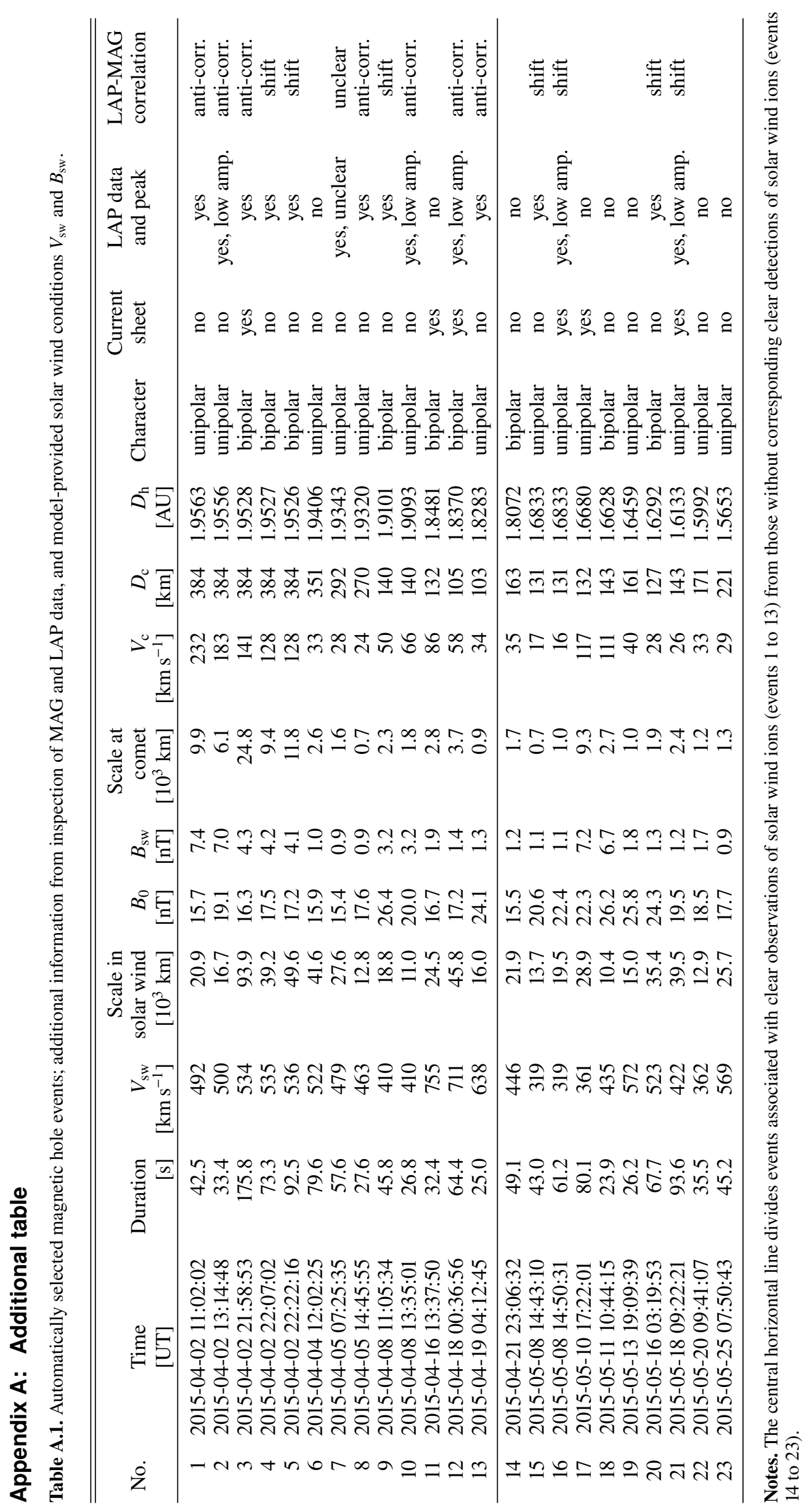

